\title{
Chalcogenide glass waveguides with paper-based fluidics for mid-infrared absorption spectroscopy
}

\author{
Vinita Mittal*, Milos Nedeljkovic, David J Rowe, Ganapathy Senthil \\ MuRugAN, JAMES S WiLKINSON
}

Optoelectronics Research Centre, University of Southampton, Southampton, SO17 1BJ United Kingdom

Received XX Month XXXX; revised XX Month, XXXX; accepted XX Month XXXX; posted XX Month XXXX (Doc. ID XXXXX); published XX Month XXXX

\begin{abstract}
We demonstrate the integration of paper fluidics with mid-IR chalcogenide waveguides to introduce liquid samples to the waveguide evanescent field for analysis. Spectroscopy of model analytes (water and IPA) having well-defined mid-IR absorptions, on a ZnSe rib waveguide fabricated on silicon, is demonstrated in the wavelength range $2.6-3.7 \mu \mathrm{m}$, showing their $\mathrm{O}-\mathrm{H}$ and $\mathrm{C}-\mathrm{H}$ stretching absorptions. The results are compared with a theoretical waveguide model, achieving good agreement. It is concluded that the presence of paper in the evanescent field does not interfere with the waveguide measurements, opening up opportunities to combine lowcost paper-based fluidics and integrated photonic technologies. (C) 2018 Optical Society of America
\end{abstract}

OCIS codes: (130.0130) Integrated optics; (230.7370) Waveguides; (280.4788) Optical sensing and sensors; (300.6340) Spectroscopy, infrared.

http://dx.doi.org/

Optical waveguides for ultra-high sensitivity surface biosensing are well-established and usually incorporate etched or molded microfluidic circuits for controlled manipulation of liquid samples and their delivery to sensing regions [1,2]. However, paper-based microfluidic technologies are of increasing importance in biochemical analysis, bringing the advantages of unpowered fluid transport, timing functionality, stored reagent incorporation, disposability and low cost [3]. Monitoring proteins, lipids and nucleic acids in biological fluids such as blood and urine is crucial to clinical diagnostics and mid-infrared (MIR) absorption spectroscopy can identify and quantify biomolecular species using their specific molecular vibrations. Optical waveguides offer enhanced surface sensitivity for low detection limits [4] and, while MIR waveguides are at an early stage of development compared with their visible and NIR counterparts, recent progress has been rapid [5-8]. Chalcogenide glass waveguides show particular promise for absorption spectroscopy of biomolecular species over the entire spectral "fingerprint" region [5,9]. In this Letter we study the incorporation of simple paper-based fluidics within the evanescent field of a chalcogenide glass optical waveguide on silicon to allow MIR absorption spectroscopy of liquid samples.

The unique physical properties of paper in terms of porosity, texture, particle retention and wetstrength allow transport of liquid analytes to detection sites with programmed en-route sample processing. Suitable papers include chromatography paper, used for molecular separation and characterized by a defined water flow rate and absorption speed, and quantitative filter paper used for filtering and gravimetric analysis, defined by average pore size [10]. These papers are generally made up of a two dimensional network of cellulose fibers, resulting in voids or pores separated by the fiber walls, in which capillary forces cause liquids to penetrate and travel along the paper [11]. Sophisticated functional microfluidic devices with microfluidic channels have been formed by wax-patterning or laser-writing in filter paper $[3,12]$. Here we demonstrate paperbased microfluidic delivery on an integrated waveguide for sensing of aqueous analytes using simple unpatterned filter paper as this is the most commonly used paper not containing additives such as fillers or clay [13]. It is expected that scattering and absorption from the paper itself will be minimal due to the weak overlap of sparse scatterers with the evanescent field, as has been observed with red blood cells [14]. This technique is expected to be more convenient than sophisticated polymer based microfluidic assemblies in many applications and may be combined with mid-IR comb, QCL or supercontinuum sources, according to the application.

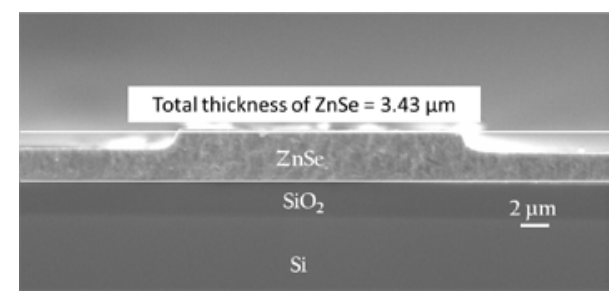

Fig. 1 SEM image of the cleaved cross-section of a ZnSe rib waveguide on oxidized $\mathrm{Si}$. 


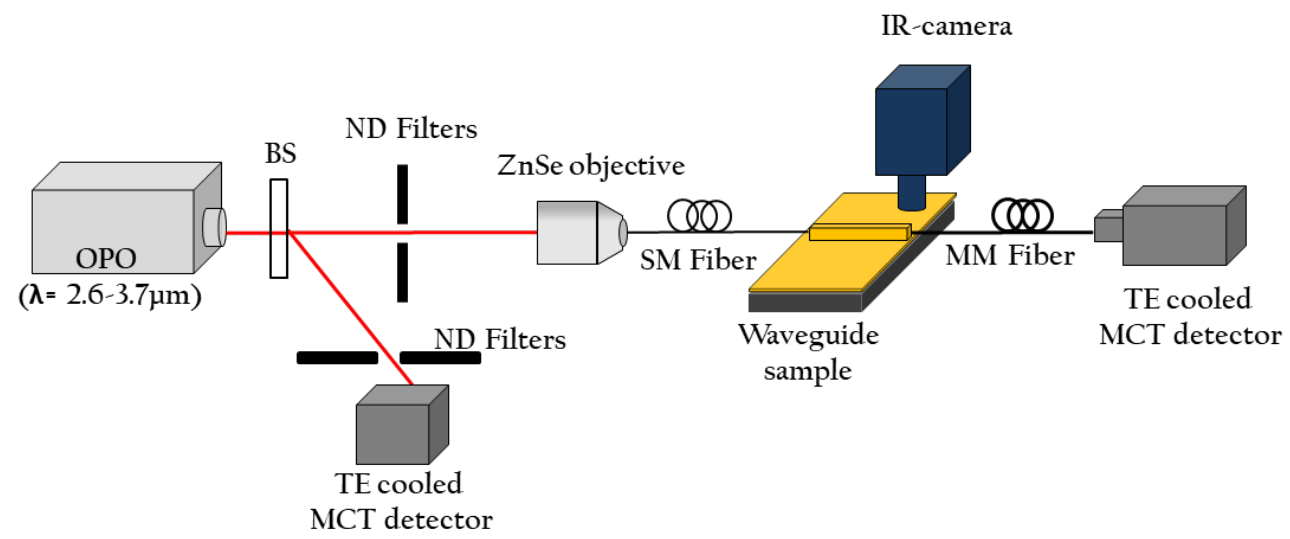

Fig. 2 Experimental apparatus for MIR waveguide spectroscopy.

To demonstrate MIR evanescent absorption spectroscopy with paper-based fluidics, ZnSe rib waveguides on oxidized Si substrates were used. An SEM image of the cleaved cross-section of such a waveguide is shown in Fig. 1. $3.4 \mu \mathrm{m}$ thick $\mathrm{ZnSe}$ films were deposited by thermal evaporation and $20 \mu \mathrm{m}$ wide rib waveguides were fabricated by photolithography and $\mathrm{Ar}^{+}$ion-beam etching to a depth of $1.23 \mu \mathrm{m}$. The end facets were cleaved to a length of $10 \mathrm{~mm}$ for fiber butt-coupling. Details of the ZnSe material deposition and waveguide fabrication can be found in [8]. Loss due to the $\mathrm{SiO}_{2}$ undercladding was simulated and predicted to be low at wavelengths up to $4 \mu \mathrm{m}$ due to the small fraction of modal power travelling in it $[15,16]$.

Waveguide spectroscopy was carried out using the apparatus shown in Fig. 2. Light from a tunable OPO (M-Squared) operating at wavelengths between 2.6 and $3.7 \mu \mathrm{m}$ was directed through a $\mathrm{ZnSe}$ objective into a single mode $\mathrm{ZrF}_{4}$ fiber (core diameter $\sim 9 \mu \mathrm{m}$ ) buttcoupled into a waveguide in the TE polarization. The waveguide output was collected using a multimode $\mathrm{InF}_{3}$ fiber (core diameter $\sim 100 \mu \mathrm{m}$ ), directed onto a calibrated TE cooled MCT detector (VIGO System). $\mathrm{A} \mathrm{CaF}_{2}$ beam-splitter (BS) was used to provide a reference signal for OPO output power and an identical calibrated TE cooled MCT detector was used to collect the power from the BS. This reference measurement was used to confirm the repeatability of incident power between waveguide measurements. The output power from the waveguide was maximized at a wavelength near 3 $\mu \mathrm{m}$ by adjusting the input and output fiber positions and then the wavelength was scanned from $2.6 \mu \mathrm{m}$ to $3.7 \mu \mathrm{m}$ with a $2 \mathrm{~nm}$ stepsize and the transmitted power was recorded on a computer. This established an analyte-free waveguide reference spectrum $\left(\mathrm{P}_{0}(\lambda)\right)$ to which output spectra with absorbing samples were normalized.

A drop of deionized (DI) water of diameter $\sim 5 \mathrm{~mm}$ was pipetted onto the waveguide at room temperature and the output power spectrum was recorded. A piece of filter paper (Fisher) covering a 3 $\mathrm{mm}$ length of waveguide was placed on the waveguide with a glass cover slip on top as shown in Fig. 3(a) and a drop of water was pipetted onto the exposed "tail" of the paper. The water was allowed to wick along the paper via capillary action and the waveguide output power spectrum was again recorded. These output spectra $\left(\mathrm{P}_{\mathrm{a}}(\lambda)\right)$ were divided by the sample-free reference spectrum $\left(\mathrm{P}_{0}(\lambda)\right)$ to yield transmission spectra for the water-covered waveguide with and without filter paper, which are shown in Fig. 3 (b) and deduced as $\mathrm{T}(\lambda)=\mathrm{P}_{\mathrm{a}}(\lambda) / \mathrm{P}_{\mathrm{o}}(\lambda)$. It was confirmed that placing the dry filter paper and cover slip in the waveguide did not observably alter the transmission spectrum. Fig. 3 (b) also shows theoretical transmission spectra of the waveguides with a water superstrate of path length $3 \mathrm{~mm}$ and $5 \mathrm{~mm}$ calculated for the fundamental mode in the TE polarization. This was calculated using finite element method modelling (COMSOL) of the complex modal effective index for the waveguide with cladding index given by the published complex refractive indices of water for wavelengths from $2.6 \mu \mathrm{m}$ to $3.7 \mu \mathrm{m}$ [17], taking dispersion fully into account.

The theoretical model used the ZnSe rib waveguide cross-section structure as fabricated, with the refractive index of $\mathrm{ZnSe}$ measured by ellipsometry [18] and that of silica taken from literature [19] including dispersion for wavelengths from $2.6 \mu \mathrm{m}$ to $3.7 \mu \mathrm{m}$. A schematic of the simulated waveguide and the fundamental mode intensity profile at $\lambda=3 \mu \mathrm{m}$ is shown in Fig. 4 . The model calculates the imaginary part of effective index of the waveguide induced by introducing a bulk absorbing medium on the surface of the waveguide, from which transmittance was derived. The complex $\mathrm{N}_{\text {eff }}$ of this mode in water at $\lambda=2.7$ and 3.0 was $2.40-\mathrm{j} 1.35 \times 10-5$ and 2.39-j3.79 x 10-4, respectively.

Agreement between experiment and theory is good both with paper and without. The most significant difference between the experimental spectra is that the spectrum with the paper in place demonstrates lower absorption because the paper covers a shorter length of waveguide than the free water drop, and this is confirmed by the simulations. Other discrepancies are minor and are probably due to mechanical misalignments between spectral measurements. It can be concluded that the introduction of paper fluidics into the evanescent field does not interfere significantly with the spectroscopic measurement. More precise definition and optimization of the sample/waveguide interaction length could in future be achieved by wax patterning of the paper. 
(a)

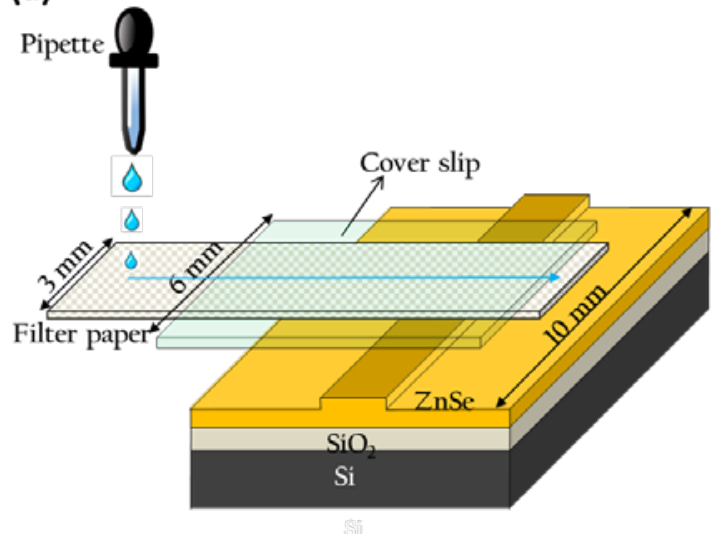

(b)

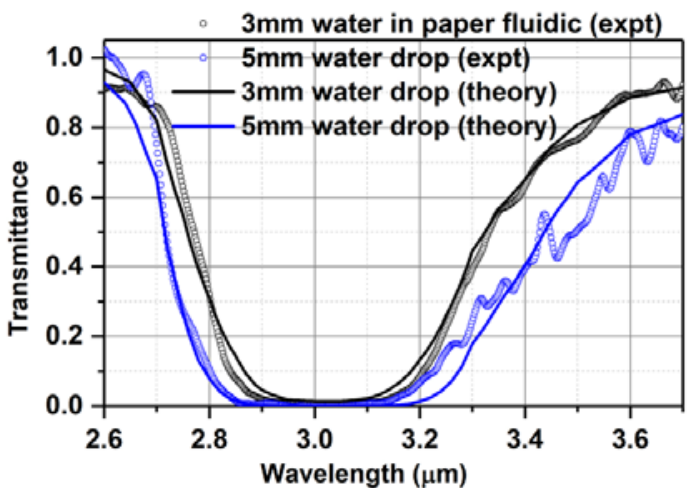

Fig. 3 (a) Illustration of the waveguide assembly used for liquid sample measurements and (b) Transmission spectra of the waveguide with water superstrate with and without filter paper, compared with theoretical predictions.

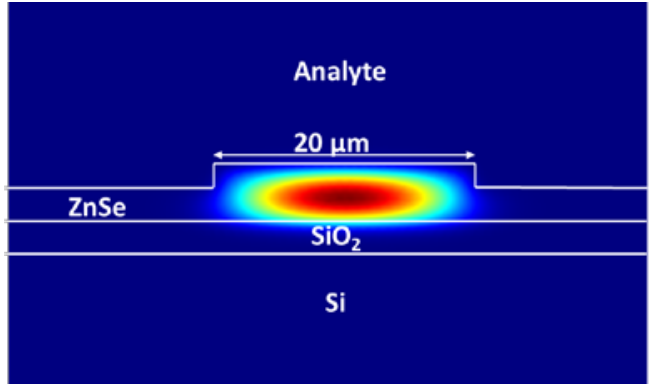

Fig. 4 Simulated geometry and mode intensity profile of a $\mathrm{ZnSe}$ waveguide with a water superstrate as analyte.

Six solutions of different concentrations of IPA (Fisher Scientific, > 99.8\% purity) in DI water were prepared in order to demonstrate spectroscopy of simple mixtures (100\% IPA, 80\%, 60\%, 40\%, 20\% IPA in water by volume and $100 \%$ water). The solutions were dropped onto the tail of the filter paper using a pipette, as before. Solutions containing IPA were refreshed while taking measurements to compensate for evaporation. A fresh filter paper was used for each measurement and all the measurements were carried out at room temperature. For each concentration, the waveguide output power spectrum with filter paper and cover slip alone $\left(\mathrm{P}_{0}(\lambda)\right)$ and with filter paper, cover slip and IPA/water solution $\left(\mathrm{P}_{\mathrm{a}}(\lambda)\right)$ was recorded, with each scan being averaged 10 times for improved noise performance. The transmittance for each solution is shown in Fig. 5. The broadband absorption at wavelengths between $2.8 \mu \mathrm{m}$ and $3.3 \mu \mathrm{m}$ is due to the $\mathrm{OH}$ vibration band present in both water and IPA. Absorption peaks corresponding to $\mathrm{CH}$ symmetric and asymmetric vibrations in IPA can be seen at wavelengths between $3.3 \mu \mathrm{m}$ and $3.5 \mu \mathrm{m}$. The $\mathrm{CH}$ absorption due to IPA is observed to weaken with reducing concentration, as expected. The wavelengths and shape of the water and IPA absorptions are in agreement with literature $[17,20]$.
To model a waveguide with IPA analyte, the published complex refractive indices of IPA [20] were used in the theoretical model described above. Fig. 6 shows the theoretical waveguide transmission spectra for pure IPA calculated using the COMSOL model as above for path lengths of 3 and $6 \mathrm{~mm}$, superimposed over the corresponding experimental spectra from Fig. 5. The theoretical and experimental spectra are in agreement with each other in terms of spectral shape, but the experimental absorptions are stronger than those predicted by theory for a path length of $3 \mathrm{~mm}$. It is proposed that this difference is due to the experimental path length of IPA on the waveguide being longer than the $3 \mathrm{~mm}$ simulated. In the case of IPA, due to its volatility and with the measurement duration of one spectrum being about 10 minutes, the analyte was refreshed continuously during a measurement. While the filter paper covered a $3 \mathrm{~mm}$ length of waveguide, the cover slip covered $\mathrm{a} \sim 6 \mathrm{~mm}$ length, and IPA may have filled the full length of the cover slip due to it being refreshed as supported by the theoretical results for a $6 \mathrm{~mm}$ path length given in Fig 6 .

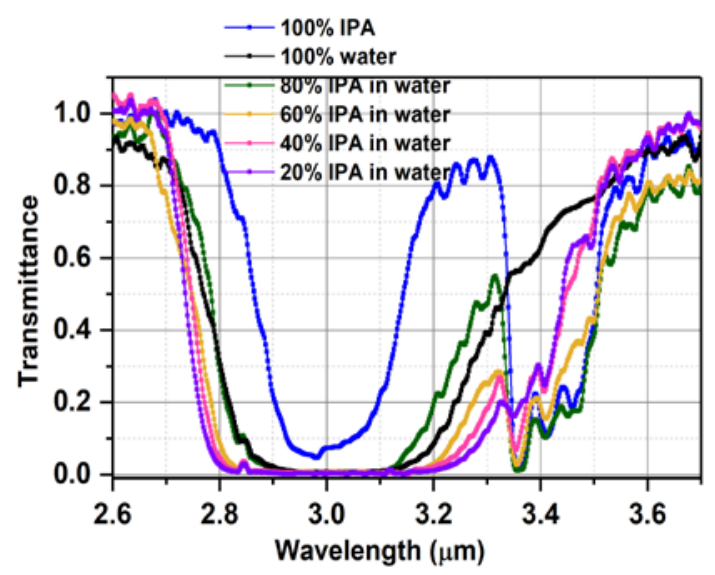

Fig. 5 MIR waveguide transmission spectra of six different concentrations of IPA in water. 


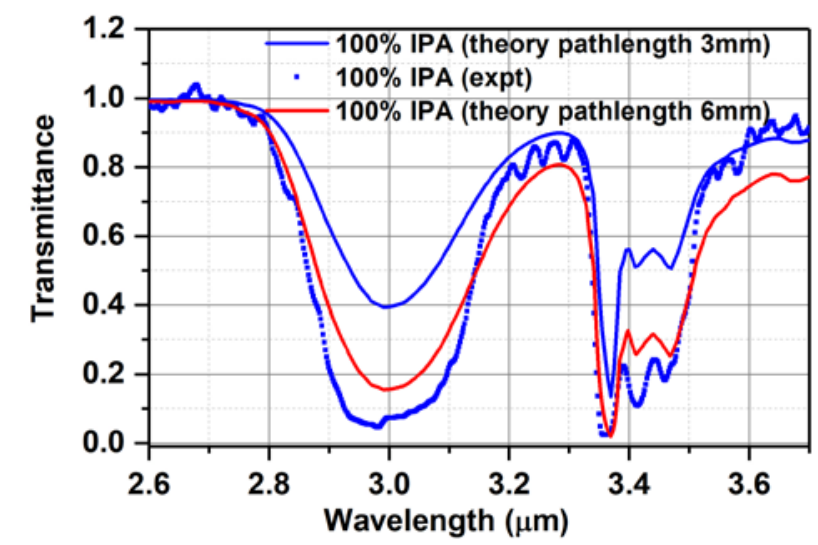

Fig. 6 Experimental and theoretical MIR waveguide transmission spectra of pure IPA on the surface of a $\mathrm{ZnSe}$ waveguide.

In this paper, the potential of using paper-based fluidics for sample delivery and analysis within the evanescent field of a ZnSe rib waveguide fabricated on oxidized Si was studied in the MIR for the wavelength region of 2.6-3.7 $\mu \mathrm{m}$. Simple filter paper was used to deliver liquid samples to the waveguide evanescent field and was found not to interfere with the waveguide transmission or the measured absorption spectrum of the analyte, despite being in intimate contact with the waveguide surface. Waveguide transmission spectra of six different concentrations of IPA in water were measured in the MIR, demonstrating the ability to analyse aqueous mixtures. Experimental spectra of waveguides with water as the analyte showed excellent agreement with theory, both with and without the paper carrier. However, the experimental waveguide absorption spectra for the samples containing IPA were found to be stronger than predicted by theory. This was probably due to spreading of the analyte away from the paper, which could be controlled in future devices by introducing printed fluidic channels in the paper. It is possible that the multimode nature of the experimental waveguide may also have contributed to the error when compared with the single-mode waveguide modelled. This work demonstrates the potential of using well-established paperbased fluidic systems in combination with highly sensitive waveguide evanescent spectroscopy approaches for low-cost sensing applications.

Funding. The authors thank the European Research Council under the European Union's Seventh Framework Programme (FP7/2007-2013) ERC grant agreement no. 291216 "Wideband Integrated Photonics for Accessible Biomedical Diagnostics" and Royal Academy of Engineering (RAEng) (M. Nedeljkovic fellowship RF201617/16/33) for funding this work.

Data supporting this paper are openly available from the University of Southampton repository at https://doi.org/10.5258/SOTON/D0499

\section{REFERENCES}

1. Z. Wang, H. Yan, S. Chakravarty, H. Subbaraman, X. Xu, D.L. Fan, A. X. Wang, and R. T. Chen, Opt. Lett. 40, 1563 (2015).

2. P. Friis, K. Hoppe, O. Leistiko, K. Bo Mogensen, J. Hübner, and J. P. Kutter, Appl. Opt. 40, (2001).

3. D. M. Cate, J. A. Adkins, J. Mettakoonpitak and C. S. Henry, Anal. Chem. 87, 19 (2015).

4. W. Lukosz, Biosens. Bioelectron. 6, 215-225 (1991).

5. G. A. Aldo, E. Baudet, L. Bodiou, J. Lemaitre, I. Hardy, F. Faijan, B. Bureau, V. Nazabal, and J.Charrier, Opt. Express 24, 23109 (2016).

6. M. Nedeljkovic, J. S. Penades, V. Mittal, G. S. Murugan, A. Z. Khokhar, C. Littlejohns, L. G. Carpenter, C.B. Gawith, J. S. Wilkinson, and G. Z. Mashanovich, Opt. Express 25, 27431 (2017).

7. V. Mittal, A. Aghajani, L. G. Carpenter, J. C. Gates, J. Butement, P. G. R. Smith, J. S. Wilkinson, and G. S. Murugan, Opt. Lett. 40, 2016 (2015).

8. V. Mittal, N. P. Sessions, J. S. Wilkinson and G. S. Murugan, Opt. Mat. Express 7, 712 (2017).

9. V. Mittal, J. S. Wilkinson, and G. S. Murugan, "Chalcogenide waveguides for mid-infrared applications," presented in EMN Meeting on Photonics, Barcelona, Spain 19-23 Sep 2016 (Invited).

10. Paulapuro, Hannu, Paper and Board Grades, Papermaking Science and Technology 18 (The Finnish Paper Engineers' Association and TAPPI, 2000).

11. D. D. Liana, B. Raguse, J. J. Gooding, and E. Chow, Sensors 12, 11505 (2012).

12. P. J. He, I. N. Katis, R. W. Eason, and C. L. Sones, Biomicrofluidics 9, 026503 (2015).

13. Sartorius Stedim Biotech, "Filter Papers for the Laboratory and Industry," https://www.sartorius.com/_ui/images/h02/hd8/881400 3290142.pdf.

14. G. Mitchell, "Absorption spectroscopy in scattering samples using integrated optics," IEEE J. Quantum Electron. 13, 173 (1977)

15. Y. Zhang, S. Yang, A. E. J. Lim, G. Q. Lo, C. Galland, T. BaehrJones, and M. Hochberg, IEEE Photon. Technol. Lett. 25, 422 (2013).

16. G. Z. Mashanovich, C. J. Mitchell, J. S. Penades, A. Z. Khokhar, C. G. Littlejohns, W. Cao, Z. Qu, S. Stanković, F. Y. Gardes, T. B. Masaud, H. M. H. Chong, V. Mittal, G. Senthil Murugan, J. S. Wilkinson, A. C. Peacock, and M. Nedeljkovic, J. Lightwave Technol. 35, 624 (2017).

17. G. M. Hale and M. R. Querry, Applied optics 12, 555 (1973).

18. V. Mittal, J. S. Wilkinson, and G. S. Murugan, "High-contrast, $\mathrm{GeTe}_{4}$ waveguides for mid-infrared biomedical sensing applications." Proc. of SPIE 8988, 89881A-1 (2014).

19. R. Kitamura, P. Laurent and J. Miroslaw, Appl. Opt. 468118 (2007).

20. E. Sani, and A. Dell'Oro, Opt. Mat. 60137 (2016). 


\section{Full References:}

1. Z. Wang, H. Yan, S. Chakravarty, H. Subbaraman, X. Xu, D.L. Fan, A. X. Wang, and R. T. Chen, "Microfluidic channels with ultralow-loss waveguide crossings for various chip-integrated photonic sensors," Opt. Lett. 40, 1563-1566 (2015).

2. P. Friis, K. Hoppe, O. Leistiko, K. Bo Mogensen, J. Hübner, and J. P. Kutter, "Monolithic integration of microfluidic channels and optical waveguides in silica on silicon," Appl. Opt. 40, 6246-6251 (2001).

3. D. M. Cate, J. A. Adkins, J. Mettakoonpitak \& C. S. Henry, "Recent developments in paper-based microfluidic devices” Anal. Chem. 87, 19-41 (2015).

4. W. Lukosz, "Principles and sensitivities of integrated optical and surface plasmon sensors for direct affinity sensing and immunosensing," Biosens. Bioelectron. 6, 215-225 (1991).

5. G. A. Aldo, E. Baudet, L. Bodiou, J. Lemaitre, I. Hardy, F. Faijan, B. Bureau, V. Nazabal, and J. Charrier, "Optical characterization at 7.7 $\mu$ m of an integrated platform based on chalcogenide waveguides for sensing applications in the mid-infrared." Opt. Express 24, 2310923117 (2016).

6. M. Nedeljkovic, J. S. Penades, V. Mittal, G. S. Murugan, A. Z. Khokhar, C. Littlejohns, L. G. Carpenter, C.B. Gawith, J. S. Wilkinson, and G. Z. Mashanovich, "Germanium-on-silicon waveguides operating at mid-infrared wavelengths up to $8.5 \mu \mathrm{m}$." Opt. Express 25, 2743127441 (2017).

7. V. Mittal, A. Aghajani, L. G. Carpenter, J. C. Gates, J. Butement, P. G. R. Smith, J. S. Wilkinson, and G. S. Murugan, "Fabrication and characterization of high-contrast mid-infrared GeTe 4 channel waveguides," Opt. Lett. 40, 2016-2019 (2015).

8. V. Mittal, N. P. Sessions, J. S. Wilkinson and G. S. Murugan, "Optical quality ZnSe films and low loss waveguides on Si substrates for midinfrared applications," Opt. Mater. Express 7, 712-725 (2017).

9. V. Mittal, J. S. Wilkinson, and G. S. Murugan, "Chalcogenide waveguides for mid-infrared applications," in EMN Meeting on Photonics, Barcelona, Spain 19-23 Sep 2016 (Invited).

10. Paulapuro, Hannu. "Paper and Board Grades, Papermaking Science and Technology, Book 18," published in cooperation with the Finnish Paper Engineers' Association and TAPPI. Jyväskylä, Finland (2000).

11. D. D. Liana, B. Raguse, J. J. Gooding, and E. Chow, "Recent advances in paper-based sensors," Sensors 12, 11505-11526 (2012).

12. P. J. He, I. N. Katis, R. W. Eason, and C. L. Sones, "Laser-based patterning for fluidic devices in nitrocellulose," Biomicrofluidics 9 , 026503 (2015).

13. Sartorius Stedim Biotech, "Filter Papers for the Laboratory and Industry," https://www.sartorius.com/_ui/images/h02/hd8/8814003290142.pdf.

14. G. Mitchell, "Absorption spectroscopy in scattering samples using integrated optics," IEEE J. Quantum Electronics 13, 173-176 (1977).

15. Y. Zhang, S. Yang, A. E. J. Lim, G. Q. Lo, C. Galland, T. Baehr-Jones, and M. Hochberg, "A CMOS-compatible, low-loss, and low-crosstalk silicon waveguide crossing," IEEE Photon. Technol. Lett 25, 422-425 (2013).

16. G. Z. Mashanovich, C. J. Mitchell, J. S. Penades, A. Z. Khokhar, C. G. Littlejohns, W. Cao, Z. Qu, S. Stanković, F. Y. Gardes, T. B. Masaud, H. M. H. Chong, V. Mittal, G. Senthil Murugan, J. S. Wilkinson, A. C. Peacock, and M. Nedeljkovic, "Germanium Mid-Infrared Photonic Devices," J. Lightwave Technol. 35, 624-630 (2017).

17. G. M. Hale and M. R. Querry, "Optical constants of water in the 200-nm to 200- $\mu$ m wavelength region," Appl. Opt. 12, 555-563 (1973).

18. V. Mittal, J. S. Wilkinson, and G. S. Murugan, "High-contrast, GeTe 4 waveguides for mid-infrared biomedical sensing applications." Proc. of SPIE 8988, 89881A-1 (2014).

19. Kitamura, P. Laurent and J. Miroslaw, "Optical constants of silica glass from extreme ultraviolet to far infrared at near room temperature," Appl. Opt. 46 8118-8133 (2007).

20. E. Sani, and A. Dell'Oro, "Spectral optical constants of ethanol and isopropanol from ultraviolet to far infrared," Opt. Mat. 60 137141 (2016). 